\title{
High-grade urothelial bladder cancer in children: A case report and critical analysis of the literature
}

\author{
Milan Kral ${ }^{\mathrm{a}}$, Jaroslav Michalek ${ }^{\mathrm{b}}$, Jozef Skarda ${ }^{\mathrm{b}}$, Tomas Tichy ${ }^{\mathrm{b}}$, Oldrich Smakal ${ }^{\mathrm{a}}$, Roman Kodetc, Vladimir Student ${ }^{\mathrm{a}}$
}

Background. Bladder cancer is relatively common in adults. In children, it is extremely rare and in the majority of cases, low grade, low stage urothelial cancers are found.

Case report. We describe the diagnostic, therapeutic, and follow-up management of bladder cancer in a 3-year-old boy examined for painless hematuria. Transurethral resection of the tumor was performed and T1 high grade urothelial cancer with osseous metaplasia was found in definitive specimens. During the 2-year follow-up, there has been no recurrence. Typical characteristics of the most prevalent bladder tumors are presented.

Conclusion. Despite its low incidence and low prevalence bladder cancer in children is a very serious condition which must not be missed in the differential diagnosis of hematuria or urinary tract infection. It is vital to differentiate urothelial cancer from hamartoma and nephrogenic adenoma and, particularly in osseous metaplasia, from sarcomatoid carcinoma. Especially in high-grade cancers, precise TUR of the tumor with a careful follow-up is essential to detect cancer recurrence and reduce progression.

Key words: bladder cancer, treatment, children, pathology

Received: June 25, 2016; Accepted: August 10, 2016; Available online: October 3, 2016 http://dx.doi.org/10.5507/bp.2016.045

${ }^{a}$ Department of Urology, Faculty of Medicine and Dentistry, Palacky University Olomouc and University Hospital Olomouc, Czech Republic ${ }^{b}$ Department of Clinical and Molecular Pathology, Faculty of Medicine and Dentistry, Palacky University Olomouc and University Hospital Olomouc, Czech Republic

'Department of Pathology and Molecular Medicine, Faculty Hospital Motol and 2nd Faculty of Medicine, Charles University in Prague, Czech Republic

Corresponding author: Vladimir Student, e-mail: studentv@seznam.cz

\section{INTRODUCTION}

Tumors of the urinary bladder are among the most serious malignant diseases of the urinary tract. These tumors represent about $5-10 \%$ of all cancers worldwide. According to Global Cancer Statistics for the year 2012, bladder cancer incidence and mortality rates are estimated to be 430,000 and 165,000 cases, respectively. Among men, the incidence is three times higher than in women ${ }^{1}$. Due to the incidence to mortality ratio in adults, bladder cancer is considered one of the most serious diagnoses in oncological urology. The most important risk factor is tobacco smoking where the risk is 2-6 times higher in smokers than in non-smokers ${ }^{2}$. From the histological point of view, transitional cell cancer (TCC) has the highest prevalence. Carcinogens causing TCC include aromatic amines (e.g., 2-naphthylamine, 4-aminobiphenyl, benzidine). The second most common type of bladder cancer is spinocellular cancer (10\%) due to chronic urinary infection caused by schistosoma haematobium. At the time of diagnosis, $70-80 \%$ of tumors are non-muscle invasive (staging Ta, T1 or carcinoma in situ). The situation in children is completely different. Urothelial malignancies are a rare presentation in pediatric patients. Like adults, transitional cell cancers are histologically the most prevalent. Of these, neoplasias of low malignant potential and low-grade tumors are present, with the tumors being non-invasive (staging pTa) $\left(\right.$ ref. $\left.^{3}\right)$. Infiltrating (pT1) or high-grade cancers are extremely rare. Painless hematuria is usually the dominant symptom indicated for further examination. Exclusion or precise assessment of the location and extent of bladder cancer requires careful cystoscopy followed by curative transurethral tumor resection (TUR). The differential diagnosis must include urinary infection, stone formation, or possibly a foreign body in the urethra or bladder.

\section{CASE REPORT}

We present the case of a 3-year-old boy examined by his pediatrician due to an isolated episode of painless gross hematuria in December 2013. During further urine testing, only mild microscopic hematuria was detected. Sonography revealed an exophytic tumor $13 \times 6 \mathrm{~mm}$ on the dorsal wall of the urinary bladder with well-marked blood supply on color-flow mapping (Fig. 1 and 2). Upper urinary tract sonography showed no pathology. The patient was indicated for cystoscopy under general anesthesia, which confirmed a 12-mm papillomatous tumor with a thin stalk on the back wall of the bladder. Transurethral resection with cold cup biopsy of the tumor bed was performed. The patient was discharged after permanent catheter removal on the second postoperative day. Specimens were sent for histopathological assessment. In accord with a second-look opinion from the pathology department at a 


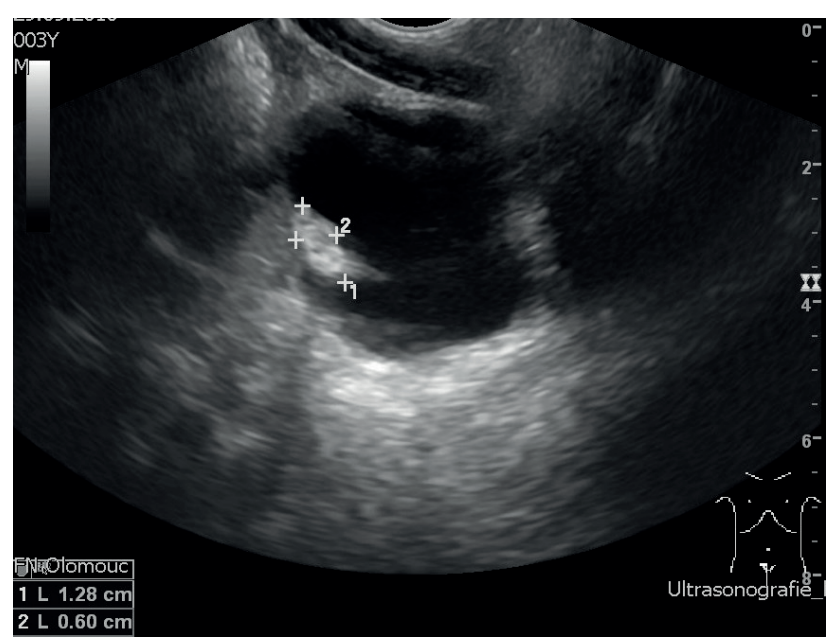

Fig. 1. Transabdominal sonography - sagital view, urinary bladder with tumour.

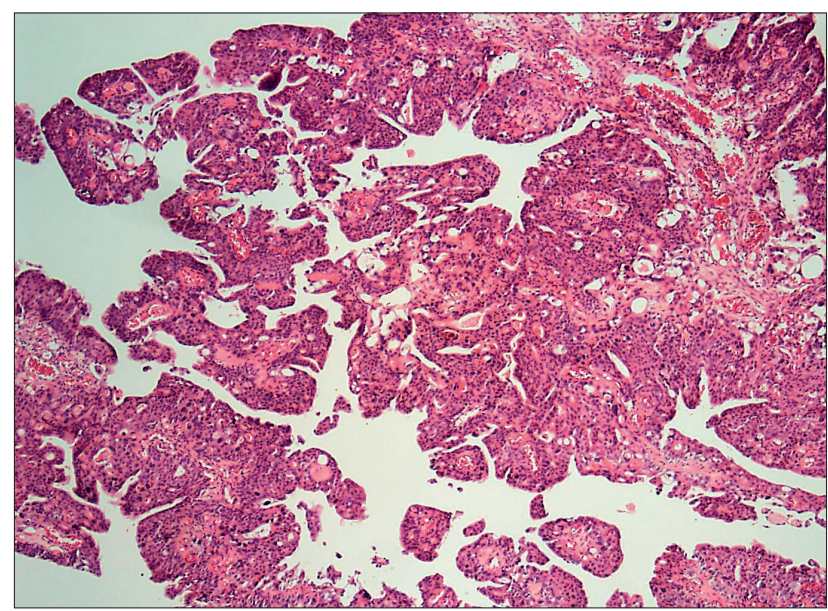

Fig. 3. Microscopy - HG papillocarcinoma (H\&E, 100x).

different university, the primary evaluation at our hospital revealed high-grade stage $\mathrm{T} 1$ transitional cell cancer with a papillary configuration according to the 2004 WHO classification of bladder tumors (Fig. 3). The papillae were lined by urothelium of various widths, with evident focal nuclear atypia. Surprisingly, glandular, squamous, and osseous metaplasias were present. Immunohistochemistry revealed positivity of p53 and aberrant expression of CK 20 in the tumor (Fig. 4). The Ki-67 index as a marker of proliferation activity was as high as $20 \%$. In the stromal papillae, metaplastic bone parts were focally present (Fig. 5).

The follow-up schedule involves regular check-ups in 3-6-month intervals: urinalysis, urine cytology, and kidney and bladder sonography. One year after the initial TUR, cystoscopy under general anesthesia was indicated, with no pathological finding in the bladder. Upper urinary tract tumor was ruled out by magnetic resonance urography (MR-urography). The recurrence-free interval has already lasted two years since the initial diagnosis.

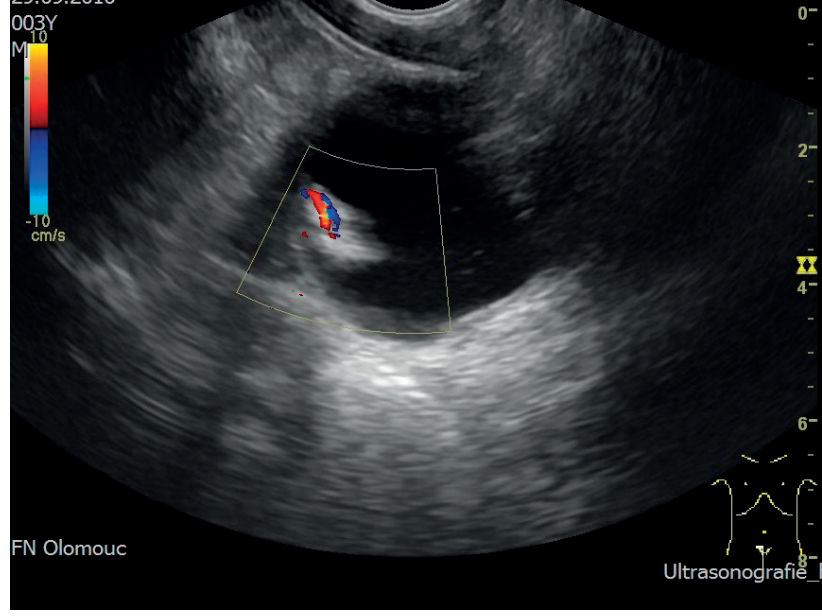

Fig. 2. Transabdominal sonography - sagital view, urinary bladder with tumour with color-flow mapping.

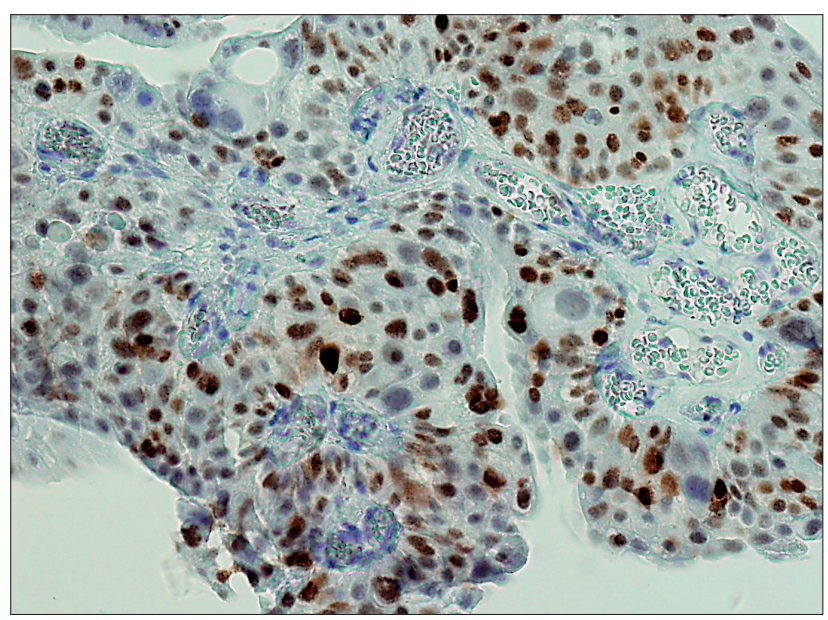

Fig. 4. Microscopy - p53 positivity in tumor cells (400x).

\section{DISCUSSION}

Urinary bladder carcinomas are tumors typical for an environmental background. The most important risk factor is smoking where smokers are more often affected at a younger age and they are often higher grade or stage. It is estimated that up to $50 \%$ of bladder cancers in men and $35 \%$ in women are directly caused by smoking. The male to female ratio is 3:1 and racial difference is also typical with a 39-fold higher prevalence among Caucasian men compared to African Americans. Transitional cancer of the bladder is a very rare malignancy in patients under 40 years (1-2.4\% of all cancers) and among patients under 20 years it is extremely rare $(0.1-0.4 \%$ of all bladder cancers) ( ref. $^{3-7}$ ). According to a recent meta-analysis, only 103 bladder cancer patients under 20 years have been described. Moreover, while two thirds of the patients were older than 15 years, only 14 patients were under 10 years of age ${ }^{3}$. A typical sign is usually painless gross hematuria, often as an isolated symptom. Sonography can reveal an exophytic bladder tumor, but cystoscopy is of crucial 


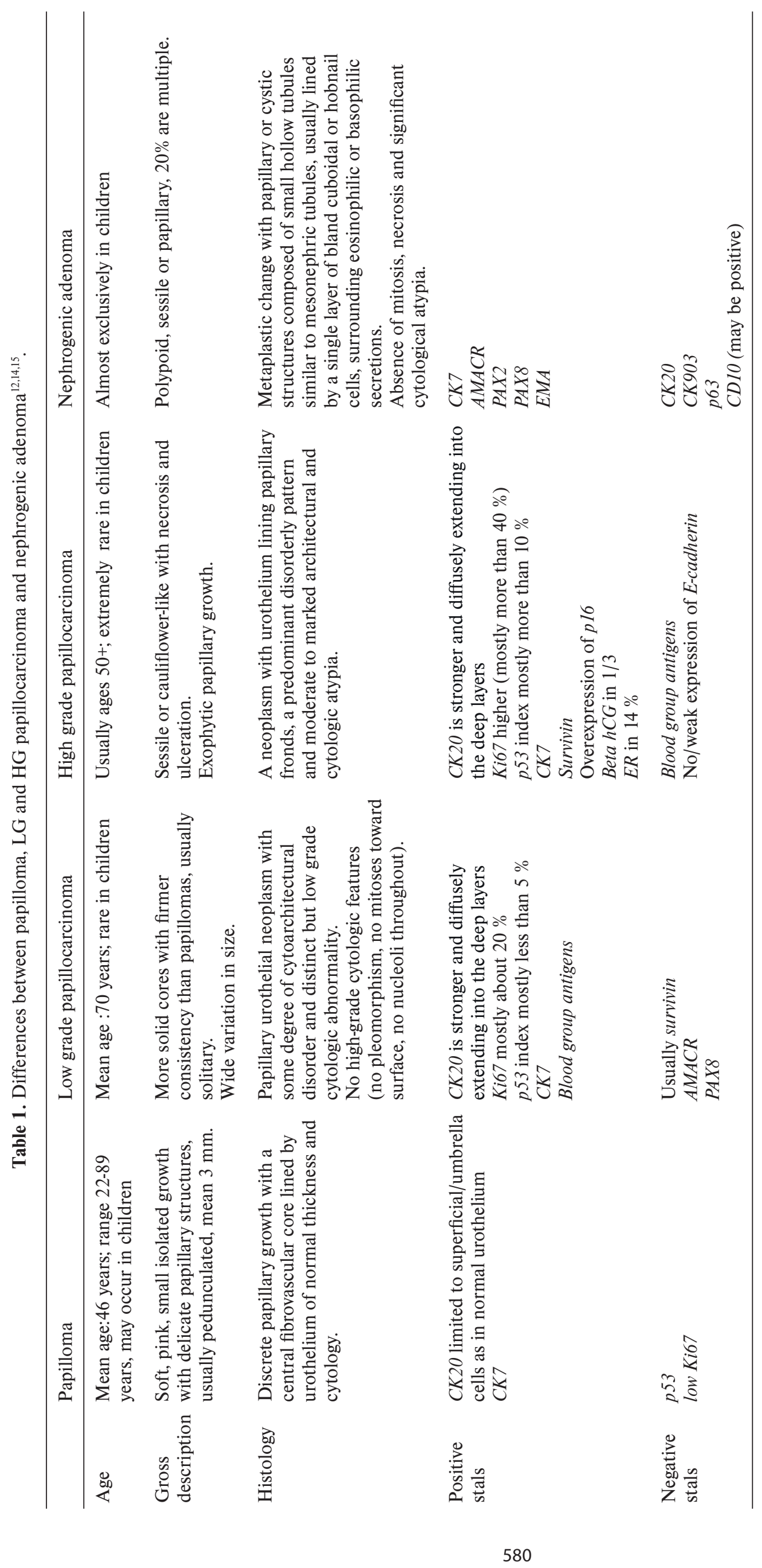




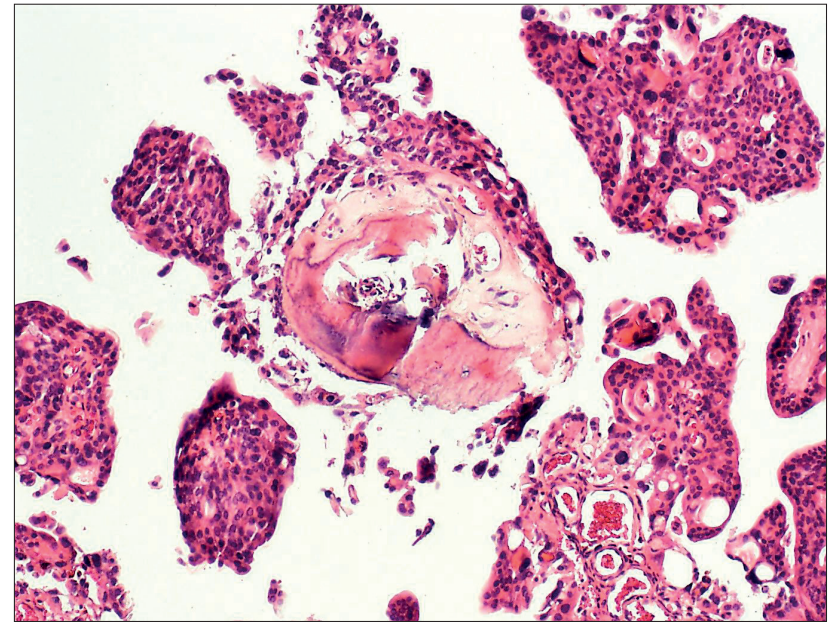

Fig. 5. Microscopy - bone metaplasia in stromal papillae (H\&E 200x).

importance with subsequent histological confirmation of the tumor by biopsy or TUR.

The configuration of the urothelial tumor is usually papillary, but it can also be solid or mixed. The lesion is often seen as a thickening of the epithelium with an increased number of cell layers evenly distributed but densely packed. In adults, urothelial cancers are non-muscle invasive (i.e., Ta and T1) in more than $75 \%$ of cases and in children the number is even higher.

While the diagnosis and treatment of bladder cancer in children/young patients is quite straightforward and routine, there is a lack of uniformity in the postoperative follow-up in terms of the extent and frequency of examinations. In adults, the schedule of postoperative cystoscopies, cytologies, and upper urinary tract examinations is an essential part of all urological guidelines (American Urological Association, European Urological Association, National Comprehensive Cancer Network etc.) (ref. ${ }^{8}$ ); however, there are no precise follow-up recommendations for children. Most follow-up protocols use sonography to exclude recurrence. Combined cytology and cystoscopy are only seldom used on a regular basis although they have a higher recurrence detection rate compared to sonography alone. The frequency and type of examination vary according to tumor risk stratification (low - intermediate - high risk). In general, the recurrence rate after initial tumor excision/resection can be as high as 40-50\%, with an additional $10-15 \%$ of progression to muscular involvement in adult patients. In children, recurrence and progression occur rarely. According to meta-analyses ${ }^{9,10}$, the recurrence rate in patients younger than 20 years is only $7 \%$, with tumor progression being exceptional. While single (or maintenance) intravesical instillation of chemotherapy is a standard and essential part of treatment after TUR, its use in children has only been described in a few cases. In contrast to the prevalent transitional cell tumors and the less frequently diagnosed spinocellular cancers or adenocarcinomas in adults, it is urothelial tumors that are exclusively detected in pediatric patients. Transitional cell carcinoma with osseous metaplasia of the stroma is a rare variant of urothelial carcinoma. In the literature, there are only a few case reports describing this condition which must be distinguished especially from sarcomatoid carcinoma ${ }^{11}$.

In children, it is particularly important to include two benign conditions in the differential diagnosis of bladder tumors: hamartoma and nephrogenic adenoma. Nephrogenic adenoma as a benign proliferation of the small glands of the urinary tract can almost exclusively be seen in the urinary bladder in children. The endoscopic appearance can mimic a malignant tumor due to the papillary appearance. Furthermore, the papillae may be mistaken for TCC on microscopy ${ }^{12}$. Bladder hamartomas are extremely rare and in children, with y few cases having been published so far. Microscopically, the glands are dilated and may be filled with eosinophilic or proteinaceous secretions. Other pathological findings include a higher vessel density and a lack of cellular atypia or mitotic activity $^{13}$. These are crucial differences in comparison with a typical urothelial bladder malignancy. Basic differences between papilloma, low grade (LG), high grade (HG) papillocarcinoma and nephrogenic adenoma are listed in Table 1.

\section{CONCLUSION}

After excluding urinary tract infection, stones or a foreign body in the urinary tract, urothelial bladder cancer has to be ruled out in children with gross hematuria. In pediatric cases, low-grade TCCs are typically found. It is important to differentiate urothelial cancer from hamartoma and nephrogenic adenoma and, particularly in osseous metaplasia, from sarcomatoid carcinoma. Especially in high-grade cancers, precise TUR of the tumor with a careful follow-up is essential to detect cancer recurrence and to reduce progression.

Author contributions: MK, VS: treatment, data analysis, manuscript preparation, final approval; OS: treatment; JM, JS: specimen revision, data analysis, manuscript preparation, final approval; TT, RK: specimen revision, data analysis.

Conflict of interest statement: None declared.

\section{REFERENCES}

1. Torre LA, Bray F, Siegel RL, Ferlay J, Lortet-Tieulent J, Jemal A. Global cancer statistics, 2012. CA Cancer J Clin 2015;65(2):87-108.

2. Parkin DM. The global burden of urinary bladder cancer. Scand J Urol Nephrol Suppl 2008;(218):12-20.

3. Paner GP, Zehnder P, Amin AM, Husain AN, Desai MM. Urothelial neoplasms of the urinary bladder occurring in young adult and pediatric patients: a comprehensive review of literature with implications for patient management. Adv Anat Pathol 2011;18(1):79-89.

4. Colombel M, Soloway M, Akaza H, et al. Epidemiology, Staging, Grading and Risk Stratification of Bladder Cancer. European Urology Supplements 2008;7:618-26.

5. Wan J, Grossman HB. Bladder carcinoma in patients aged 40 years or younger. Cancer 1989;64:178-81. 
6. Alanee S, Shukla AR. Bladder malignancies in children aged $<18$ years: results from the surveillance, epidemiology and end results database. BJU Int 2009;106:557-60.

7. Lerena J, Krauel L, García-Aparicio L, Vallasciani S, Sunol M, Rodó J. Transitional cell carcinoma of the bladder in children and adolescents: Six-case series and review of the literature. J Pediatr Uro 2010;6:481-5.

8. http://uroweb.org/wp-content/uploads/EAU-Guidelines-Nonmuscle-invasive-Bladder-Cancer-2015-v1.pdf

9. Berrettini A, Castagnetti M, Salerno A, Nappo SG, Manzoni G, Rigamonti W, Caione P. Bladder urothelial neoplasms in pediatric age: experience at three tertiary centers. J Pediatr Urol 2015;11(1):26. e1-5.
10. Javadpour N, Mostofi FK. Primary epithelial tumors of the bladder in the first two decades of life. J Urol 1969;101:706-10.

11. Mege-Lechevallier F, Cherasse A, Ronze S, Colombel M, Scoazec JY. Transitional carcinoma of the ureter with osseous metaplasia of the stroma: a case report. 2007;27(1):43-6.

12. Sathe PA, Ghodke RK, Kandalkar BM. Multifocal nephrogenic adenoma - a mimicker of malignancy. Indian J Pediatr 2012;79(12):1661-3

13. Ota T, Kawai K, Hattori K, Uchida K, Akaza H, Harada M. Hamartoma of the urinary bladder. Int J Urology 1999;6:211-4.

14. Safaei A, Farzaneh MR, Amin Sharifi AR. Immunohistochemistery Study in a Case of Nephrogenic Adenoma of Bladder. Iran J Med Sci 2012;37(2):137-40.

15. www.pathologyotlines.com/bladder 